\title{
Multimodal MRI Techniques and Their Importance in Frameless Navigation Biopsy for Brain Glioma
} \author{
Saba Firouznia, ${ }^{1,2}$ Parto Sabetrasekh, ${ }^{3}$ and Alireza Ahmadian ${ }^{2,4, *}$ \\ ${ }^{1}$ Faculty of Mechanical Engineering, Amir-Kabir University of Technology, Tehran Polytechnic, Tehran, Iran \\ ${ }^{2}$ Research Center for Biomedical Technology and Robotics, Tehran University of Medical Sciences, Tehran, Iran \\ ${ }^{3}$ Advanced Diagnostic and Interventional Radiology Research Center, Imam Khomeini Hospital, Tehran University of Medical Sciences, Tehran, Iran \\ ${ }^{4}$ Department of Medical Physics and Biomedical Engineering, Tehran University of Medical Sciences, Tehran, Iran \\ "Corresponding author: Dr. Alireza Ahmadian, Department of Medical Physics and Biomedical Engineering, Research Center for Biomedical Technology and Robotics, Tehran \\ University of Medical Sciences, Tehran, Iran. E-mail: ahmadian@tums.ac.ir
}

Received 2017 August 01; Accepted 2017 September 12

Keywords: Multimodal, MRI Techniques, Frameless, Navigation Biopsy, Brain, Glioma

Gliomas represent $86 \%$ of primary intracranial malignant tumors. Their most malignant form, glioblastoma multiform (GBM), is either astrocytic, oligodendrocytic, or includes a variety of other histo-pathological subtypes (1). The best non-invasive imaging modality for neurooncologic evaluation of the patients is MRI, which is used for differential diagnosis, biopsy guidance, therapeutic planning, and follow-up.

Although conventional MRI is used for diagnosis, neurosurgical treatment planning, and follow up, current developments in this field, including MR spectroscopy (MRS), perfusion MRI, and diffusion-weighted imaging (DWI) have been used with acceptable success in these patients (1).

The most common form of brain edema, which is associated with brain tumors, is vasogenic edema that shows high T2 signal intensity. The non-enhancing region of the lesion that surrounds the enhanced tumor is referred to as peritumoral infiltrative edema (2).

Gliomas have an infiltrative manner, and usually invade the surrounding tissues microscopically beyond the area of the enhancing tumor. Therefore, in glioma, the peritumoral edema is a combination of reactive non-tumoral edema and infiltrative edema due to altered capillary morphology and tumor cell infiltration. As the specificity of conventional MRI in differentiating tumor infiltration in the peritumoral edema is limited, advanced MRI techniques are used to evaluate physiological and metabolic alternations (3).

Current modalities in MRI techniques including MR spectroscopy (MRS), diffusion-based techniques, and MR perfusion have been used to evaluate the peritumoral area.

The only MRI method that gives information on water diffusion is DWI which uses phase-defocusing and phaserefocusing gradients to assess the rate of microscopic water diffusion within the tissues. This technique has been used to evaluate the grade or to differentiate brain tumors according to cellularity (4).

Cerebral tumors with higher cellularity may show a remarkable decrease in apparent diffusion coefficient (ADC) values, which hypothetically could depict neoplastic cell infiltration areas in the peritumoral region (5). It seems that mean or minimum ADC values can remark neoplastic cell infiltration in the peritumoral region.

MRS offers a reliable method for definite and exact brain tumor diagnosis by implementing biochemical information that is mainly related to mitotic cell division (total choline compounds [Cho]), neural tissue displacement (N-acetyl-aspartate [NAA]), energy metabolism (total Creatine [ $\mathrm{tCr}]$ ), and necrotic transformation (lipids [Lip]) (6).

MRS can also predict the response to therapy, recognize the borders between viable tumor and brain parenchyma depicted on conventional MRI, and discriminate between tumor tissue and post radiation necrosis. MRS and conventional MRI together can increase the diagnostic accuracy in brain tumor detection (6).

Representing a depiction of a dynamic pathophysiologic process, perfusion MRI, according to its image acquisition method, is mainly divided into three categories (1). First, in dynamic susceptibility contrast MRI (DSC-MRI), after injection of a bolus of paramagnetic material (i.e. gadolinium-based contrast media), a rapid measurement of T2-weighted signal change is applied. Then, 5-10 minutes after gadolinium contrast media injection, dynamic contrast enhanced MRI (DCE-MRI) is created on the basis of T1-weighted signal changes. 
Following these steps, arterial spin labelling (ASL), which principally uses water as a freely diffusible endogenous tracer of blood entry in specific imaging volumes, has insufficient efficacy for clinical applications.

Using these techniques, measurement of peculiar factors such as cerebral blood volume (CBV), cerebral blood flow (CBF), and microvascular permeability parameters are possible.

DSC-MRI and DCE-MRI have been used to obtain relative cerebral blood volume ( $\mathrm{rCBV}$ ), which is typically measured by calculating CBV parameter, relative to contralateral normal brain tissue. DCE MRI is also able to evaluate microvascular permeability by measuring trans endothelial leakage of gadolinium-based contrast.

Perfusion MRI has been used to identify WHO tumor grade, tumor genotyping, and biopsy guidance in gliomas $(1,7)$. rCBV and microvascular permeability increase with the increase in tumor grade. However, in mixed gliomas this may be inaccurate (8).

MRI and advanced MRI protocols are rapidly becoming the main routine in cerebral tumor imaging techniques to improve the diagnosis of brain lesions.

In clinical evaluations, advanced MRI protocols are used to complement the morphological findings from anatomical MRI by metabolic and functional information. Therefore, combination of MRI protocols could significantly and non-invasively improve the diagnostic accuracy for differentiating between tumor-infiltrating edema and vasogenic edema (2).

As the best treatment method for patients with gliomas requires assessment of the histopathological diagnosis, a specific and reliable histopathological diagnosis is very important. Image fusion by super-imposing MRI and CT scan results in meticulous definition of target points, and also reduces complications.

Till the 1980s, frame-based stereotaxis was the standard technique for precise localization of small intracranial lesions by introducing catheters into the tumors (9).

Considered as the gold standard method for inspecting intracranial lesions, frame-based techniques, mostly apply a rigid-frame, which provides the best targeting precision (10). However, patient discomfort, prolonged surgical time and the risk of postoperative infection at the fixture points of the frames, are some limitations of this technique.

The advent of image-guided surgical procedures has provided enormous advances in the field of neurosurgery. The idea behind using frameless computer-aided biopsy in navigation systems is real time depiction of the position of the needle tip in the corresponding images without requiring a stereotactic frame as well as its easy to use and comparable diagnostic yield (10). The major approach of the navi- gation system is to use advanced methods for analysis and reconstruction of the information produced from prevalent medical imaging modalities such as MRI and CT scans on one hand and the three dimensional (3D) position information of surgery tools from accurate noncontact tracking systems on the other hand.

Frameless and frame-based stereotactic-biopsy techniques apply preoperative images with a registered probe to access the target tissue. Indeed they have a similar disadvantage that there is no real-time radiographic feedback confirming the needle in the target tissue.

The surgical navigation system improves accuracy and reduces intervention time, morbidity, and intensive care and hospital costs. Today, IGS is used to help surgeons plan the surgery by providing accurate information about the anatomy, and it also enables minimally invasive interventions, since the intraoperative images can be used interactively as a guide.

Frameless systems have no need to attach an uncomfortable frame to the head of an awake patient for imaging and intervention with comparable accuracy with framebased systems (11).

The principle process of frameless navigation depends on the spatial registration of anatomic landmarks in a 3D model according to the reconstruction of cross-sectional images. Furthermore, frameless stereotaxic biopsy is safe and effective in collecting diagnostic samples in different regions of the brain (12). Although stereotactic biopsy systems rely on preoperative images, navigation-based preoperative images may cause an unacceptable error degree due to deformation of tissues during surgery or deep penetration of tool tips that subsequently decrease the efficiency of biopsy.

The reported failure rate of stereotactic biopsy is approximately $7 \%-15 \%$ due to sampling errors (6). Sample error commonly occurs when the selected target is in a necrotic center of a tumor or the enhancing area is missed. Therefore, a high-grade glioma might be misdiagnosed as a low-grade glioma. This problem should be resolved by the proposed registration and fusion of multimodal MRI images, which precisely locate the necrotic center and other areas of the tumor. This will enable the IGS system to guide the biopsy tool towards the desired location of tumor and perform a complete resection. Meticulous preoperative analysis of MR images, selection of multiple targets for biopsy and using multimodal imaging can obviate these difficulties.

As mentioned previously multimodal imaging including MRS, DWI and perfusion imaging have great advantages in providing additional diagnostic information about metabolism and biochemistry of gliomas. Notably, these techniques are noninvasive methods that support 
target selection in gliomas during image-guided navigation procedures (13).

Finally, information from advanced imaging techniques combined within the reference frame of these frameless navigation biopsy systems can be helpful in predicting the location of the lesion as well as vital structural and functional neighboring regions that may be at risk during biopsy procedures.

\section{References}

1. Jenkinson MD, Du Plessis DG, Walker C, Smith TS. Advanced MRI in the management of adult gliomas. Br J Neurosurg. 2007;21(6):550-61. doi: 10.1080/02688690701642020. [PubMed: 18071982].

2. Lee EJ, Ahn KJ, Lee EK, Lee YS, Kim DB. Potential role of advanced MRI techniques for the peritumoural region in differentiating glioblastoma multiforme and solitary metastatic lesions. Clin Radiol. 2013;68(12):e689-97. doi: 10.1016/j.crad.2013.06.021. [PubMed: 23969153].

3. Pavlisa G, Rados M, Pavlisa G, Pavic L, Potocki K, Mayer D. The differences of water diffusion between brain tissue infiltrated by tumor and peritumoral vasogenic edema. Clin Imaging. 2009;33(2):96-101. doi:10.1016/j.clinimag.2008.06.035. [PubMed: 19237051].

4. Yamasaki F, Kurisu K, Satoh K, Arita K, Sugiyama K, Ohtaki M, et al. Apparent diffusion coefficient of human brain tumors at MR imaging. Radiology. 2005;235(3):985-91. doi: 10.1148/radiol.2353031338. [PubMed: 15833979].

5. Kono K, Inoue Y, Nakayama K, Shakudo M, Morino M, Ohata K, et al. The role of diffusion-weighted imaging in patients with brain tumors. AJNR Am J Neuroradiol. 2001;22(6):1081-8. [PubMed: 11415902].
6. Setzer M, Herminghaus S, Marquardt G, Tews DS, Pilatus U, Seifert $\mathrm{V}$, et al. Diagnostic impact of proton MR-spectroscopy versus imageguided stereotactic biopsy. Acta Neurochir(Wien). 2007;149(4):379-86. doi: 10.1007/s00701-007-1126-0. [PubMed: 17380247].

7. Jenkinson MD, Smith TS, Joyce KA, Fildes D, Broome J, du Plessis DG, et al. Cerebral blood volume, genotype and chemosensitivity in oligodendroglial tumours. Neuroradiology. 2006;48(10):703-13. doi: 10.1007/s00234-006-0122-z. [PubMed: 16937145].

8. Xu M, See SJ, Ng WH, Arul E, Back MF, Yeo TT, et al. Comparison of magnetic resonance spectroscopy and perfusion-weighted imaging in presurgical grading of oligodendroglial tumors. Neurosurgery. 2005;56(5):919-26. discussion 919-26. [PubMed: 15854239].

9. Khoshnevisan A, Allahabadi NS. Neuronavigation: principles, clinical applications and potential pitfalls. Iran J Psychiatry. 2012;7(2):97-103. [PubMed: 22952553].

10. Lu Y, Yeung C, Radmanesh A, Wiemann R, Black PM, Golby AJ. Comparative effectiveness of frame-based, frameless, and intraoperative magnetic resonance imaging-guided brain biopsy techniques. World Neurosurg. 2015;83(3):261-8. doi:10.1016/j.wneu.2014.07.043. [PubMed: 25088233].

11. Orringer DA, Golby A, Jolesz F. Neuronavigation in the surgical management of brain tumors: current and future trends. Expert Rev Med Devices. 2012;9(5):491-500. doi:10.1586/erd.12.42. [PubMed: 23116076].

12. Air EL, Leach JL, Warnick RE, McPherson CM. Comparing the risks of frameless stereotactic biopsy in eloquent and noneloquent regions of the brain: a retrospective review of 284 cases. $J$ Neurosurg. 2009;111(4):820-4. doi: 10.3171/2009.3.JNS081695. [PubMed: 19408980].

13. Yao C, Lv S, Chen H, Tang W, Guo J, Zhuang D, et al. The clinical utility of multimodal MR image-guided needle biopsy in cerebral gliomas. Int J Neurosci. 2016;126(1):53-61. doi: 10.3109/00207454.2014.992429. [PubMed: 25539452]. 